\title{
Microwave Manipulation of Cold Molecules
}

\author{
Interactions between molecules can be tuned using microwaves, a finding \\ that could be leveraged for studying quantum systems.
}

\section{By Katherine Wright}

$\int$ Itracold molecules are a promising platform for studying quantum many-body problems and quantum chemistry, as well as for building a quantum computer, among other things. But for all these applications, researchers require methods to manipulate the interactions between molecules. Now Zoe Yan at the Massachusetts Institute of Technology and her colleagues demonstrate a method that uses microwaves to tune the dipolar interaction between two sodium potassium ( $\mathrm{NaK}$ ) molecules [1].

The team cooled a gas of NaK molecules to $560 \mathrm{nK}$, which placed the molecules in their lowest energy state. In this state the molecules have no dipole moment, and they only notice each other's presence if two molecules happen to collide, which happens rarely. The team then applied a microwave field to the gas with a frequency close to that of the transition frequency between the molecule's ground and a particular excited state. This field "dressed" the molecules, stretching out their electric fields such that the field of each molecule roughly resembled

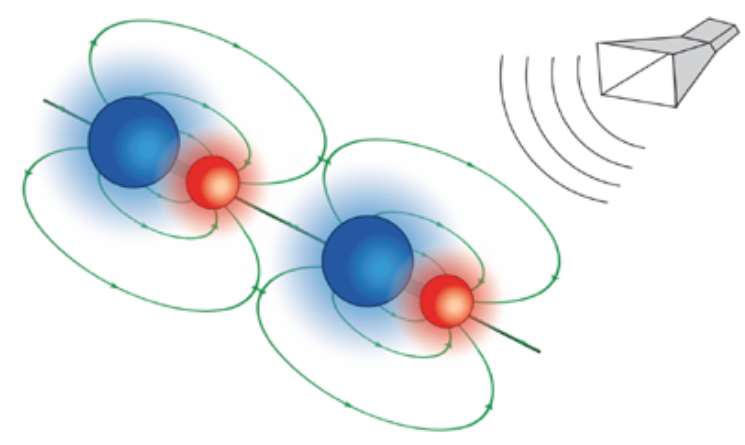

Credit: Z. Yan/Massachusetts Institute of Technology that of the magnetic field around a tiny bar magnet. This change induced strong attractive interactions between the molecules, leading to a substantial increase in the molecule collision rate.

Yan says that their method could also be used to induce repulsive interactions between molecules. Such interactions could shield the system from two-body collisions, events that can significantly limit the lifetime of molecular gases. The molecules' interactions could also be tuned to create exotic forms of matter using ultracold gases, such as topological superfluids.

This research is published in Physical Review Letters.

Katherine Wright is a Senior Editor for Physics.

\section{REFERENCES}

1. Z. Z. Yan et al., "Resonant dipolar collisions of ultracold molecules induced by microwave dressing," Phys. Rev. Lett. 125,063401 (2020). 